\title{
IPTV Home Networking via 802.11 Wireless Mesh Networks: An Implementation Experience
}

\author{
Firat Birlik, Özgür Gürbüz, Member, IEEE and Ozgur Ercetin, Member, IEEE
}

\begin{abstract}
Wireless multimedia home servers are the next generation of home entertainment systems. From a single broadband connection entering home, multimedia data is transmitted to $T V$ and other peripherals using only wireless links. The provision of hiqh quality time-critical multimedia services in indoor environment is very challenging due to high attenuation and multi-path fading caused by the walls and the contention in the shared wireless channel. In this paper, we demonstrate that mesh networking can help improve the service quality of both multimedia and data users, when the video packets are relayed with EDCA priorities based on their importance. Our results on a real wireless mesh network implementation using the new high definition video streaming standard, H.264 verify our hypothesis, where we observe almost no degradation video quality, and very low packet delay and loss rate in the presence of high amount of competing data traffic. ${ }^{l}$
\end{abstract}

Index Terms - IEEE 802.11, wireless mesh networks, wireless video, H.264, Quality of Service.

\section{INTRODUCTION}

High quality television broadcasts are transmitted over satellite, cable or ADSL-based broadband Internet connections. Internet Protocol Television (IPTV) is a relatively new and emerging alternative service that transmits TV signals over broadband Internet. In IPTV service, operators install a network switch at the customer's broadband connection, and install Ethernet cables from the switch to television sets. The wireless access points (APs) seem as a convenient drop-in replacement for the switch and all the connecting cables; however, regular IEEE 802.11 APs cannot usually satisfy transmission quality requirements of multimedia applications. Wireless video transmission via 802.11 APs is fragile against background data traffic, e.g., web browsing, and the transmission rates may vary according to the location of the user and the structure of the house. Multiple APs can be used as repeaters to provide high rate links throughout the house, but this requires additional planning by the customer or the operator. Since 802.11-based multi-hop wireless networks implement Distributed

\footnotetext{
${ }^{1}$ This work was in part supported by Airties Wireless Networks (AirTies), based on funding from Turkish Technology Development Foundation and Scientific and Research Council of Turkey. Any opinions expressed herein do not necessarily reflect those of the AirTies. Any and all intellectual property rights expressed herein are the property of AirTies except the author's rights.

F. Birlik is with Airties Wireless Networks, Istanbul, TURKEY, (e-mail: firat@airties.com).

O. Gürbüz and O. Ercetin are with the Faculty of Engineering and Natural Sciences, Sabancı University, Istanbul, 34956, TURKEY (e-mail: \{ogurbuz, oercetin\}@sabanciuniv.edu).
}

Contributed Paper

Manuscript received June 3, 2009
Coordination Function (DCF) for medium access, an end-toend route may contain several relay nodes contending for the channel resulting in degradation of video quality due to increased end-to-end delay and packet loss probability. All of these problems have prevented the replacement of indoor cabling by 802.11 links until now.

Our proposed approach aims to improve the home multimedia experience by providing high quality video streaming via wireless mesh technology. Our automatic mesh backbone helps with self-organization capability to establish and maintain high quality links between APs and provide alternate routes without user intervention. In addition to mesh implementation, we adapt and implement packet prioritization strategies into wireless mesh nodes so as to enable streaming of DVD or better quality media to any location in the house. Hence, we overcome both the coverage and contention problems by combining wireless mesh networks (WMNs) with a smart prioritization scheme.

In prior work, 802.11 based WMNs have been mainly investigated for extending wireless transmission range and for providing higher end-to-end throughput [1]. Although WMNs extend coverage and improve the quality of the point-to-point links in the network, contention is also increased in the wireless channel due to 802.11 's inherent access mechanism. Increasing contention causes increased number of packet losses and delay jitter; both of which are especially harmful for video. In [2], Enhanced Distributed Channel Access (EDCA) is presented as one of the enhancements to 802.11 to provide Quality of Service (QoS) via different priority traffic classes with different contention window sizes and Arbitration Inter-Frame Space (AIFS) values. Video quality can be improved by assigning video packets to higher priority classes. However, using the same EDCA class for all video packets is not sufficient to solve the contention problem in the network, especially when there are several video streams competing for the channel. In [3-6] the authors aim to improve the video quality over wireless transmission by designing layer-3 solutions or modifications in the video encoder. In [7], the new H.264 encoder is considered and a solution is proposed for improving the quality of streaming H.264 media over single-hop 802.11 wireless networks.

In this work, we make use of the characteristics of H.264 video encoding/decoding (codec) standard [8] to implement smart prioritization together with mesh networking. We consider H. 264 since it is a recent technology that is increasingly employed by consumer media products, high definition movie players and HDTV broadcasts [9]. H.264 is also the strongest candidate for IPTV operators as it supports high quality video with decreased bandwidth requirements. H.264 packetizes a video stream into several frame types of 
different importance levels in constructing the images. In our smart EDCA implementation, H.264 packets are analyzed and more important video packets are mapped to higher priority EDCA classes, while packets with less important content are given lower priorities. By such a mapping, the delay and loss probability of important packets remain low and this significantly improves the end-to-end video quality over multiple hops, as compared to a basic prioritization scheme where all video packets are given the same high priority.

Our experiments show that our smart prioritization scheme not only helps improve the contention in the network, but also preserve the bandwidth provided to the background data traffic. A major advantage of our approach is that the smart prioritization algorithm is implemented in the mesh nodes, embedded only in a video classifier that interfaces the wireless driver and MAC, whereas prior works in [3-6] and [7] also require changes in the encoding application and the network layer. Another contribution of our work is the illustration and performance analysis based on tests on a real-time IPTV (H.264) video streaming application over a multi-hop mesh network. Our tests prove that high quality end-to-end video streaming can be provided in homes with the commercial-offthe-shelf wireless equipment using our mesh and smart prioritization implementation, with almost no loss in Peak Signal-to-Noise Ratio (PSNR), low delay and very few packet losses in the presence of high competing data traffic.

\section{Preliminaries}

\section{A. IEEE 802.11 Essentials}

IEEE 802.11 specifies $D C F$ as the default mechanism for medium access control [10]. DCF is based on CSMA/CA, where nodes transmit if the channel is idle and they back off a random amount of time if the channel is busy, so as to avoid possible collisions with other nodes also waiting for channel becoming idle. The back-off duration is a random number of slots, where the random number is an integer number Contention Window ( $\mathrm{CW}$ ) picked from the set $\left[\mathrm{CW}_{\min }\right.$, $\left.C W_{\max }\right]$. In DCF, nodes get an equal chance of capturing the channel, and hence they observe similar Quality of Service (QoS).

IEEE 802.11e standard specifies the multimedia extensions on 802.11, one of which is named as Enhanced Distributed Coordination Access (EDCA), which provides QoS differentiation [2]. EDCA employs four access categories, "Best Effort" (BE), "Background" (BG), "Video" (VI) and "Voice" (VO) with varying priorities. Differentiated channel access is obtained through different settings of $C W$ parameters that control the random back-off durations. If a node is assigned low $C W$ parameters, it will statistically pick a shorter back-off duration, which results in a higher chance (hence, higher priority) of obtaining the channel. Voice and video classes have highest priorities followed by best effort and background traffic. EDCA does not promise any QoS guarantees, but it only partitions the available channel resources according to the access priorities. In a high contention environment, each access category suffers from the low resources, and the available resource is shared among the active transmitters proportional to their priorities.

IEEE 802.11s task group was formed for defining the primitives for 802.11-based wireless mesh networks. According to the draft standard [1], mesh capable devices are referred to as Mesh Points (MPs), which detect and establish links with each other over mesh links (ML). MPs discover paths between communicating peers through a routing algorithm. Although different routing algorithms are allowed by the standard, all nodes are required to support a mandatory routing algorithm, named Hybrid Wireless Mesh Protocol (HWMP), which is virtually a combination of AODV and tree-based routing [1]. 802.11s also introduces power and security primitives for proper operation, as well as mechanisms for congestion control and channel selection for improving throughput. At the time of development for this work, 802.11s standard was still being discussed and a draft was not ready, therefore we designed simple 802.11 based mesh networking functions which can be easily implemented using the commercial-off-the-shelf 802.11 equipment. In this work, we specifically consider the home networking application for mesh networking, hence we make use of not all but some features of $802.11 \mathrm{~s}$, such as AODV. We also specifically consider video streaming and propose prioritization schemes to work with EDCA for streaming of H.264 video across our WMN implementation.

\section{B. H.264 Video Encoding Standard}

A video codec involves a set of algorithms that encode and decode a digital video stream. We consider the H.264 codec for its widespread implementation on consumer devices including video telephony, mobile phones and portable storage media such as Apple Ipod [9]. H.264 codec can provide very high quality pictures at almost half of the bit rate of previously existing standards. The video quality is improved by optimizing network transmission according to the requirements of the codec by the help of two independent layers: 1) Video coding layer (VCL) that is responsible for compression of raw video and generation of the output video slices; 2) Network abstraction layer (NAL), which is responsible for generating the video frames and packets to be transferred. Partitioning is an extension for NAL, which divides video frames into network packets with different priorities.

Five different types of video frames are generated by H.264: Parameter Set Concept $(P)$ frames contain the encoding parameters such as picture size, display window, macro block allocation map, etc. It is essential that $P$ frames are transmitted in a reliable way, since decoding of all frames depends on $P$ frames. Instantaneous Decoding Refresh $(I)$ frames contain a coded picture that can be decoded without needing any other frame. Partition $A$ frames contain interframe motion vectors, which depend on both previous and subsequent frames, whereas Partition $B$ and $C$ frames contain only texture related information. Multiple consecutive Partition $A, B$ and $C$ frames depend on $I$-frames, and loss of a single $I$-frame may lead to successive failures. Loss of $A$ 
frames may result in multiple erroneous frames in the decoded video, but loss of $B$ and $C$ frames only decreases the image quality.

C. Challenges of Streaming Video over Wireless Mesh Networks

In a video streaming system, the receiving clients perform a real-time operation, which requires decoding and displaying frames at a specific frame rate. The original video is assembled out of the received packets considering dependencies and packet deadlines. Before discussing the challenges of video streaming over a multi hop WMN, we review the problems of video streaming over a single hop 802.11 wireless link.

In 802.11 based systems, higher layer protocols for data transmission employ greedy algorithms that aim to maximize active channel usage. However, video traffic requires a specific amount of bandwidth and if the data traffic of a neighbor tries to acquire all of the available bandwidth, then both flows are going to be suppressed. As an example, consider a hypothetical channel with maximum available throughput of $20 \mathrm{Mbps}$, to be shared between two users with traffic load of $5 \mathrm{Mbps}$ video and $20 \mathrm{Mbps}$ data, respectively. When the channel is shared equally, both flows will suffer a packet loss of $20 \%$, which may have a devastating consequence for the video flow perhaps resulting in completely halting the decoding process. Another problem of 802.11 links is the high delay jitter, i.e., high variance of the delay. In order to decode a video stream properly, video packets have to be received within their specific deadlines. Any late packets are eventually dropped at the receiver, and the corresponding frame is decoded without those packets.

Both problems are exacerbated in WMNs due to increased contention associated with increased hop count, and the processing/queueing delays imposed by each intermediate mesh node. In a WMN, nodes at the center of mesh network may relay more packets than the mesh nodes at the periphery. Therefore, transmission buffers of the nodes at the center of the topology may hold more packets than those at the periphery, causing an increase in the network delay. Intelligent resource allocation among existing flows should be investigated, so that the packet losses and delays can be bounded and sufficient quality can be provided for video flows across the WMN.

\section{Proposed PaCket Prioritization Schemes}

In order to accomplish high quality multi-hop wireless video transmission over WMNs, the required end-to-end throughput should be sustained, and the cumulative delay jitter should be minimized. Assigning video packets a higher priority than the rest of the packets, results in shorter back-off durations, and hence, lower delay jitter. In order to ensure that the required end-to-end throughput is available for video, video traffic should be resilient to the suppression by the data traffic. This is not an easy task, since there is no bandwidth allocation mechanism within 802.11 standards. Nevertheless, as demonstrated in this paper, by using intelligent prioritization of packets, the loss, delay and jitter of video packets can be decreased, which in turn minimizes the quality degradation of the decoded video at the expense of decreased throughput of background data traffic. However, this decrease is tolerable (unless the available resource is extremely scarce) as demonstrated in our experimental results.

\section{A. Basic Prioritization}

As indicated earlier, EDCA defines four access categories with varying priorities. In the basic prioritization scheme, each video packet is assigned to one of the higher priority EDCA classes, which is either Video (VI) or Voice (VO), and data traffic is assigned to a lower priority class. Video packets are transmitted with high priority in each intermediate node across the WMN. In order to achieve this, all mesh nodes should be able to classify video packets, and relay them with the predefined EDCA class, which is effectively carried out in our implementation.

\section{B. Smart Prioritization}

The basic prioritization scheme treats all video packets with the same priority. However, H.264 codec has a network abstraction layer (NAL), which creates network packets with varying importance. In a severe contention environment and across multiple hops, video packets may also get dropped despite their high priority. The dropped packets may belong to important $P$ or $I$ frames and the video quality at the receiver would be significantly reduced.

Another problem imposed by basic prioritization is the accumulated video packet contention. In single hop transmissions, video packets contend only with data traffic by maintaining a high chance of success. In WMNs, however, video packets also contend with each other. Consider an example, with $11 \mathrm{Mbps}$ links and video is to be delivered over 3 -hops and all nodes are in the same contention domain. Assuming video traffic rate of $3 \mathrm{Mbps}$, the effective contending video traffic becomes 9 Mbps, generating contentions among video packets as well. When there is contention among high priority traffic, consecutive collisions occur, the delay and jitter on video packets increase, which in turn effectively causes packets missing their delivery deadlines.

In smart prioritization, we differentiate video packets according to their importance by assigning them into different priority EDCA classes. Specifically, in our implementation, $I$ frames are assigned to VO class, $P$ and $A$ frames are assigned to VI class, and $B$ and $C$ frames are assigned to BE class of EDCA. Consequently, in case video packets get dropped, lower priority video packets are dropped first, and the most important I or P frames are preserved for last. Our smart prioritization scheme also regulates the contention in multi hop networks as the number of packets contending for each priority class is significantly reduced. Essentially using smart prioritization, the video packets with higher importance are protected more strictly than the ones with the less importance, and the end-to-end video packet loss, delay and jitter are kept under control, in order to prevent the degradation of the quality of the video. 


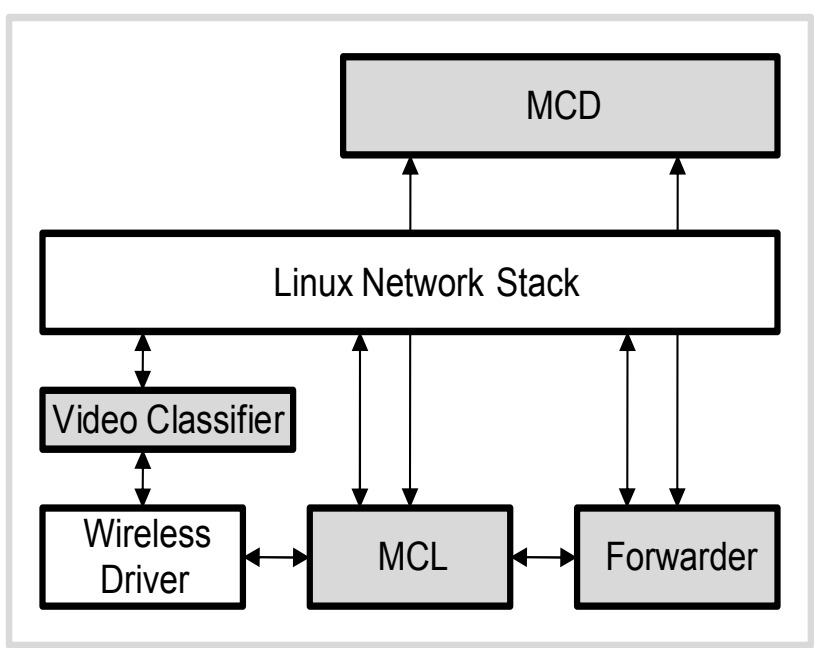

Fig. 1 General Mesh Node Architecture

\section{IMPLEMENTATION OF H.264 VIDEO OVER WMNS}

An important contribution of our work is a real demonstration with promising results, where our proposed prioritization algorithms and a wireless mesh network are implemented on commercial-off-the-shelf wireless equipment. In this section, we provide the details of this implementation.

\section{A. Architecture Overview}

Our mesh implementation has three major functions, mesh node (neighbor) discovery, mesh link establishment and routing. The general mesh node architecture is illustrated in Fig. 1. The shaded parts in the figure, represent our mesh layer implementation extensions. Mesh Control Daemon (MCD) handles the decisions for mesh node discovery, mesh link establishment, monitoring link qualities, neighbor failures and route discovery over the Mesh Control Layer (MCL). MCD also performs auto-healing functions in the mesh network in case of link failures. As a link or node failure is detected, route error messages are sent to neighboring links for notification. If any route is broken, a new route is determined using route discovery if necessary. Meanwhile, MCL is responsible for providing a logical control communication medium for MCD, using the underlying wireless card driver.

In a WMN, mesh nodes require a means of communication with each other to handle mesh operations dictated by the MCD. For this purpose, we have defined a subtype of 802.11 management frames, named as MESH_MANAGEMENT frames. We have also defined different types of mesh management frames, such as PEER_REQUEST, PEER_RESPONSE, REPLY_CODE, etc., to be identified by the subtype field within the payload, and to be used for different mesh functions as described in the subsequent section.

\section{B. Mesh Functions}

Neighbor discovery is performed via listening to the beacons. Each mesh capable AP includes a special information element in beacons that is only recognizable by other mesh capable APs, while clients and other non-mesh capable 802.11 equipment safely ignore this element. Mesh information element includes a "MESH-ID" field to differentiate different mesh networks residing in the same area. If the MESH-ID field within the mesh information element matches the mesh identifier of the receiving node, then both nodes determine that they are in the same mesh network. If both parties are part of the same mesh network, they should create a mesh link for data transmission. The first peer that has detected other node sends a PEER_REQUEST packet. The remote party can accept or deny this connection request. In both cases, the remote party sends a PEER RESPONSE packet with a PEER OK, PEER DENY or a PEER ERROR code. If a PEER OK code is received, the mesh link is created and routing layer immediately starts using this link in the routing decisions. If the PEER_REQUEST packet is not answered, it is decided that the packet is lost, and it is retransmitted periodically until a predefined timeout occurs. There is also the possibility that both parties detect each other and send PEER_REQUEST packets within a very small amount of time. This might create a deadlock where both parties will wait for a PEER_RESPONSE packet indefinitely. For conflict resolution, we have designed the PEER_REQUEST packets to contain a 32-bit random number. In a deadlock, the lower numbered request is ignored by both parties as if it was never sent and the valid request is repeated as if it was initiated for the first time.

Mesh nodes may possess different mesh capabilities. Both the mesh information element included in the beacons and other mesh management frames are designed to contain mesh capability elements indicating routing algorithms, routing metrics and other implementation or version specific features. This feature prevents incompatible mesh nodes trying to create mesh links.

The maintenance of the mesh links is based on the monitoring of the status of the remote peers. If a remote peer is not responsive, a mesh node tears down the current mesh link and notifies the routing layer about the change in the topology. If a remote peer returns to life, the link is created once again with a new peer handshake.

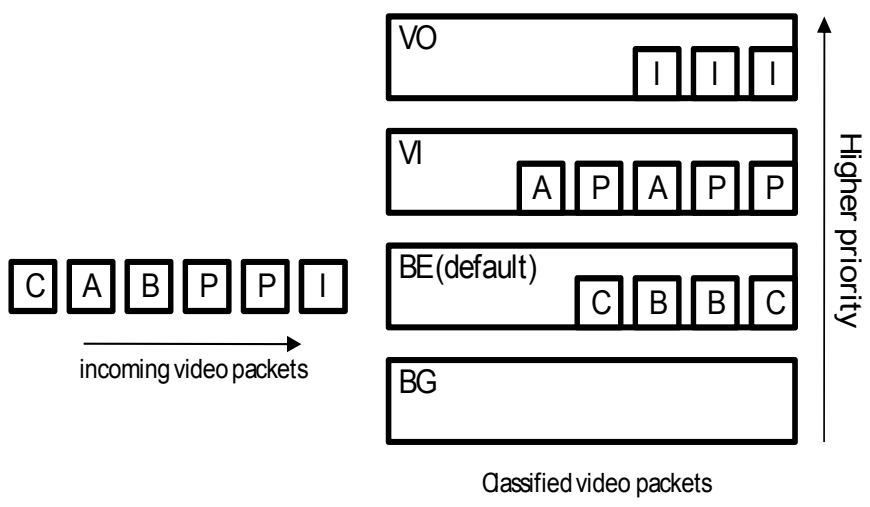

Fig. 2 Smart Prioritization for H.264 packets

We have implemented a modified version of Ad-Hoc on demand routing protocol (AODV) [11] as the routing algorithm. Packet forwarding is done by the Forwarder module, which performs layer-2 routing of packets to appropriate links that are either uplink Ethernet connections or 


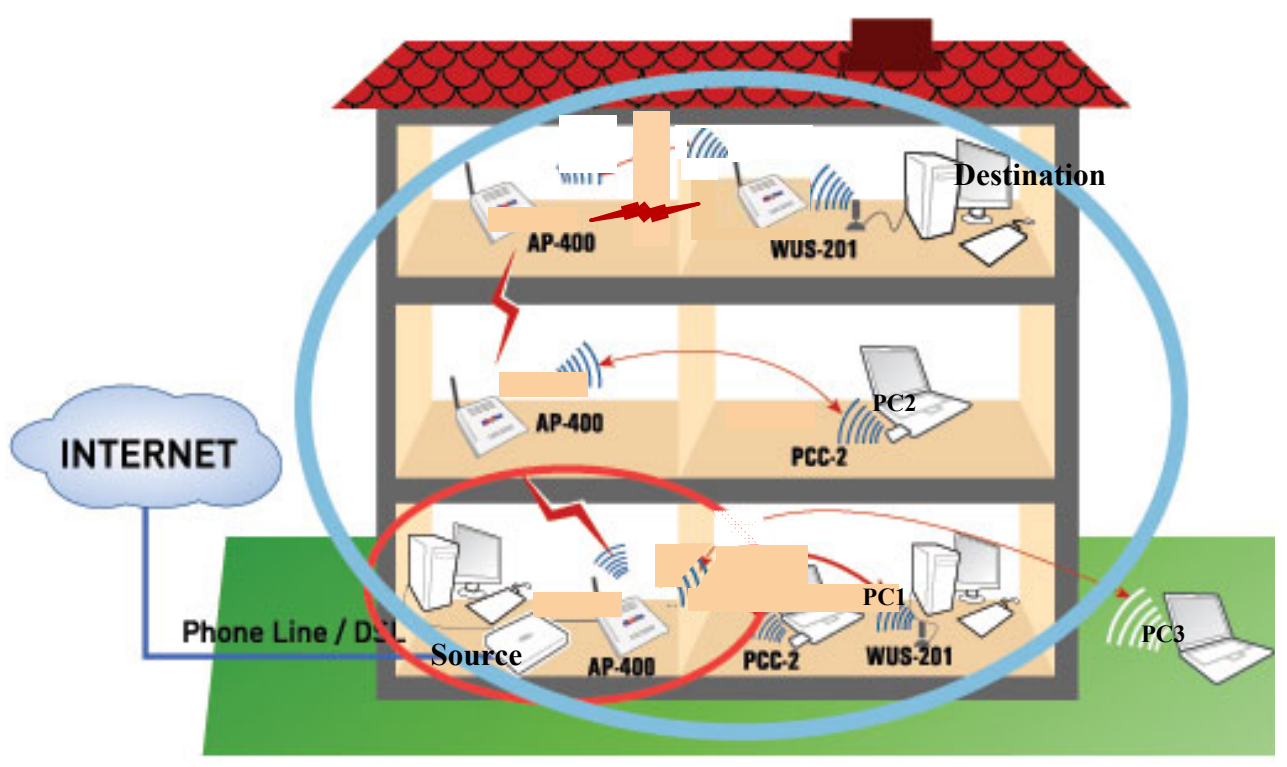

Fig. 3 Testbed Scenario

other established mesh links. The main difference of our protocol from AODV is that packets are routed according to their MAC addresses instead of IP addresses. In other words, our mesh node is identical to a layer-2 switch for clients. With this approach, we guarantee that any IP-based protocol can be supported. Another difference is that routing packets are transmitted in the form of MESH_MANAGEMENT packets instead of regular data packets, which reduces the probability of loss for those routing packets. Routing metric is left as a programmable field to adapt different routing solutions.

\section{A. Video Classifier}

In order to transmit video packets with appropriate access categories, we designed and implemented a packet inspection and classification engine, namely Video Classifier to work between the network driver and the network stack of the Linux kernel, as depicted in Fig. 1. When a packet is released from the network stack for transmission, it is captured by the Video Classifier, and it is inspected by comparing it with known codecs and packetization techniques. If the packet is identified as an H.264 NAL unit packet, it is processed further to detect the packet type, and it is passed to the driver with a priority tag determined according to Basic or Smart prioritization scheme. For basic prioritization, all video packets are enqueued in the same high priority queue, VI or VO. The queuing implementation with respect to Smart prioritization algorithm assigns different types of video packets different priorities according to their importances as shown in Fig. 2.

\section{Test Results}

We have implemented the mesh networking functions together with both the basic and smart prioritization algorithms on $802.11 \mathrm{~g}$ APs. In our implementation, we used APs with $8 \mathrm{MB}$ memory and $180 \mathrm{MHz}$ system-onchip MIPS CPU. An Atheros based wireless chipset, which fully supports EDCA in hardware level, and six distinct hardware queues are used. One of the queues is assigned for beacon transmission while other four queues are assigned to four EDCA classes. As the wireless driver, we used a highly modified version of proprietary Atheros LSDK 5.0.28 driver. The operating system is based on Linux 2.4.x kernel with an updated and recent network stack. In full functional idle state with no traffic flowing, the system has about $200 \mathrm{~Kb}$ free memory. Although our APs have limited processing power and memory, they are able to function properly under heavy traffic.

Fig. 3 depicts our test scenario, which has been set up within different floors and rooms of a house. There are four APs forming a mesh to relay the video traffic from the source node at the ingress by the ADSL modem to the far most destination node. The source and destination nodes are connected to the APs with wired links and the sensing range of the source node is marked as shown. The computers $\mathrm{PC} 1, \mathrm{PC} 2$ and $\mathrm{PC} 3$ are also communicating with each other, creating background data traffic that interferes with video. During our test runs, routing remained static for multi-hop routes between APs to ensure that any delay or packet drop due to the routing layer is prevented and only the effects of multi-hop relaying and background data traffic are investigated. H.264 video streams are encoded at the ingress of the network and decoded at the destination. All results are averaged over five different test runs, and the ambient noise/interference level remains approximately the same during these runs. 


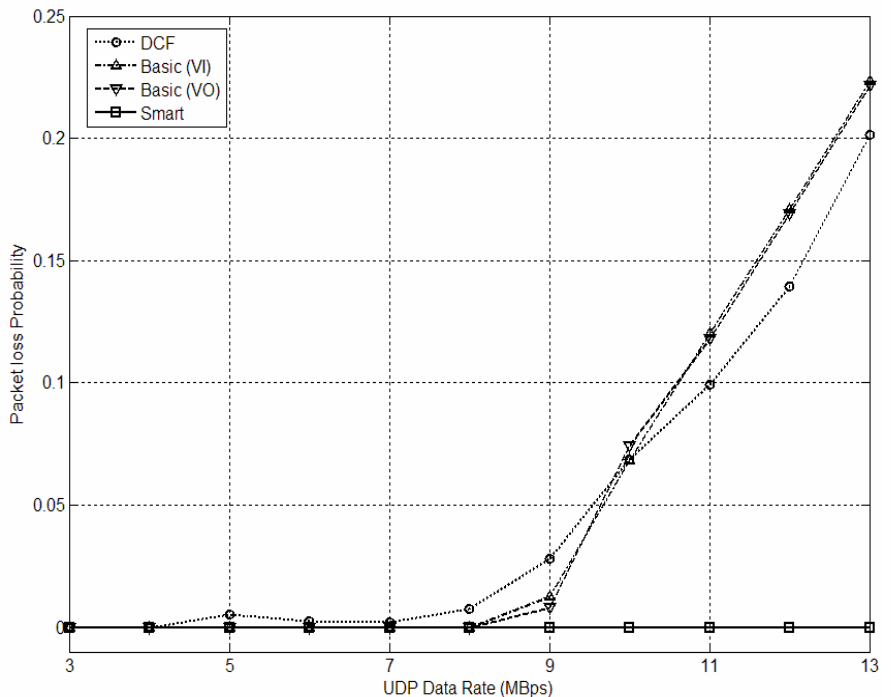

Fig. 4 Video packet loss probability vs. UDP data traffic load

In our tests, we analyzed the performance of video streams under varying traffic conditions, as we investigate the performance of DCF, and the basic and smart prioritization schemes. We also consider two variations of basic prioritization scheme, where all video packets are either classified as VO or VI EDCA classes. Data traffic is always sent at the lowest priority class. We tested video streaming performance against varying amounts of background UDP traffic. The video is streamed at approximately $3 \mathrm{Mbps}$ and the total channel capacity is about $14 \mathrm{Mbps}$. If no packet is lost, video consumes $9 \mathrm{Mbps}$ alone over three hops. The theoretical upper limit of the residual capacity is about $5 \mathrm{Mbps}$.

In Fig. 4, the video packet loss probability is plotted with respect to the contending data traffic load. If contending data traffic reaches the virtual upper limit of 5Mbps, DCF medium access scheme starts losing video packets. However, in basic and smart prioritization schemes, data traffic is suppressed, and there is no video packet loss until data traffic reach $8 \mathrm{Mbps}$. Hence, entire video is transferred without losing any information. When data traffic exceeds $8 \mathrm{Mbps}$, the basic prioritization scheme starts losing video packets, whereas the smart prioritization scheme prevents losses in video packets until data traffic reaches $13 \mathrm{Mbps}$.

According to Fig. 5, all transmission schemes including DCF have similar end-to-end mean delay until the background data traffic is $8 \mathrm{Mbps}$. When background data traffic exceeds 8 Mbps, both of the basic prioritization schemes enter a rapidly increasing delay pattern until $10 \mathrm{Mbps}$ of data traffic. In the meantime, both DCF and smart prioritization do not experience any rapidly increasing delay. However, unlike smart prioritization scheme, the main reason the packet delay of DCF is not increasing is due to the large loss of video packets, while successfully transmitting only a few.

Fig. 6 shows the Cumulative Distribution Function (CDF) of the video packet delay under two limiting cases: when background data traffic is turned off and on at the highest load level (13 Mbps). Without the background traffic, all algorithms perform similarly.

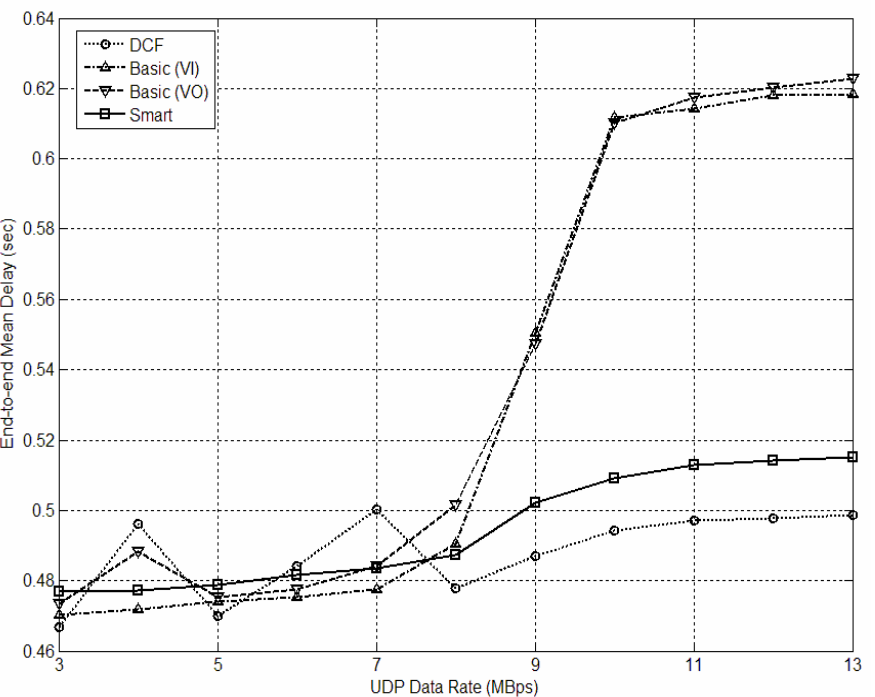

Fig. 5 Video delay vs. UDP data traffic load

Still, the basic schemes experience the lowest delay, which is due to the fact that all of the video packets are sent with high priority, with lower back off. When background data traffic is on, the picture is reversed. We observe that video packets transmitted with the two basic prioritization schemes experience higher end-to-end delay with larger jitter as compared to DCF and smart prioritization. Since all video packets are enqueued in the same buffer, they are subject to high contention and low service rate. In smart prioritization on the other hand, despite the interference of data traffic, video packets experience low contention, high service rate, resulting in stable buffers. Hence, there is no dramatic increase in delay and the variance is still low. The CDF curve for DCF involves the results for the few surviving video packets, since most packets are dropped rapidly. Also, our unreported test results indicate that DCF experiences much higher delay jitter than the other schemes, for all data traffic load levels.

Next, in order to evaluate the video quality, we have considered Peak Signal to Noise Ratio (PSNR) loss of the decoded video, which can be readily measured at the H.264 decoder. PSNR loss is defined as the difference between the PSNR value of the original and decoded video. In Fig. 7, the PSNR loss for different schemes is depicted with respect to varying background data traffic. Consistent with the packet loss probability and delay plots given in Fig. 4-6, with DCF, the video quality immediately drops by approximately $9 \mathrm{~dB}$ when the channel capacity is reached, and it further drops by $20-25 \mathrm{~dB}$ when total traffic is increased beyond the channel capacity. Both of the basic prioritization schemes do not suffer from significant quality degradation until the data traffic is $8 \mathrm{Mbps}$, while the smart prioritization observes no quality degradation till the end of the test.

An interesting and also somewhat counter-intuitive result is that basic prioritization schemes suffer to a great extent from rapidly increasing delay and packet loss. This is actually because of the fact that the transmission buffers of the intermediate nodes get full and packets are delayed or dropped in the buffers. 

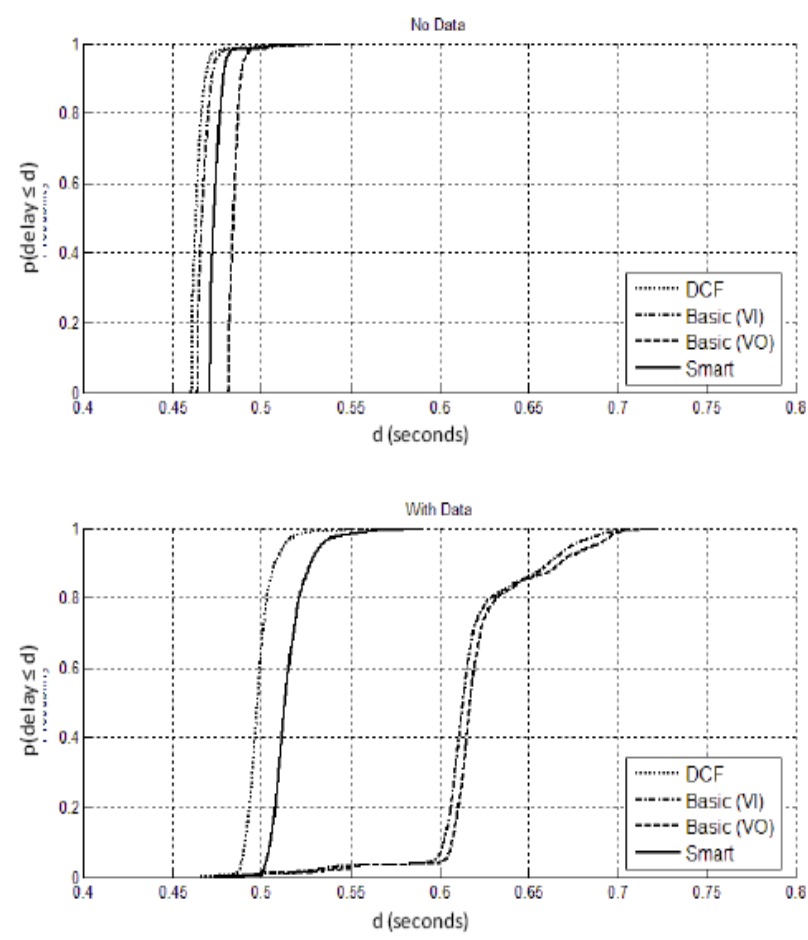

Fig. 6 CDF of video delay in the absence and presence $(13 \mathrm{Mbps})$ of UDP data traffic

The APs used in the test have only $200 \mathrm{~KB}$ of free memory, which accommodates up to 50 packets. In the basic prioritization scheme, all video packets contend with each other with the same priority. Thus, with high background data traffic, the number of packets waiting in these queues increase faster than the effective service rate, which in turn causes video packets to miss their deadlines and get dropped. In smart prioritization, video packets are distributed to different queues. Since each queue has a different priority, the packets in each queue contend only with each other. Therefore, buffers on each high priority class remain stable, where enqueue rate is smaller than the dequeue rate.

\section{CONCLUSION}

WMNs make the wireless video transmission over 802.11based networks more complicated. Because of the extra delay and packet drops imposed by multi-hop transmissions, the basic 802.11 DCF transmission scheme is not appropriate to support video transmission. For video transmission over WMN, the basic EDCA prioritization makes sense if large buffers are available on mesh nodes. However, if the resources are scarce, the basic prioritization scheme may suffer from high delays and packet loss under heavy background data traffic conditions. With our proposed smart prioritization algorithm, we overcome the additional delay and packet drop probabilities, while requiring very small buffer sizes on the APs. The background data traffic is suppressed, but the utilization of the shared channel is much higher than the basic prioritization scheme, since there are fewer collisions.

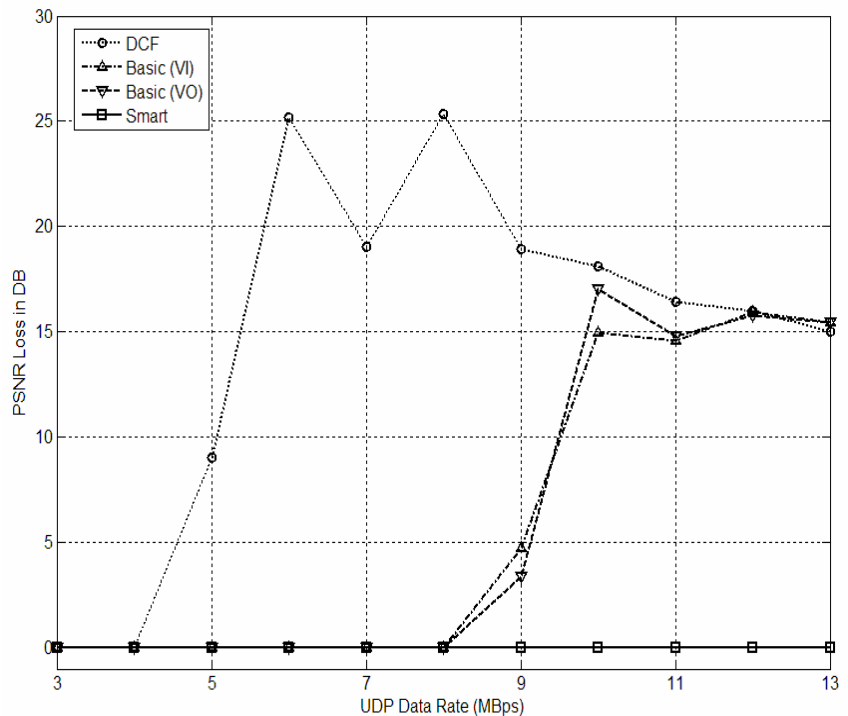

Fig. 7 Video PSNR loss vs. UDP data traffic load

There are still some remaining issues about video transmission using WMN that prevents adoption of this technology by actual IPTV service operators. In real IPTV services, video streams are transmitted using multicast packets, which are transmitted as broadcast packets with a fixed low bitrate in the wireless medium. Another practical problem is the lack of 802.1q Virtual LAN support in WMNs and especially with integrated multicast support. IPTV service operators segment their network using 802.1q VLAN's for billing purposes. Before these problems and further unforeseen problems are solved, wireless video transmission will not be able to be deployed widely in IPTV services.

\section{REFERENCES}

[1] Hiertz, G.R.; Max, S.; Rui Zhao; Denteneer, D.; Berlemann, L., "Principles of IEEE 802.11s," Proceedings of 16th International Conference on Computer Communications and Networks 2007. pp.1002-1007, Aug. 2007.

[2] "IEEE Standard for Information technology - Telecommunications and information exchange between systems - Local and metropolitan area networks - Specific requirements Part 11: Wireless LAN Medium Access Control (MAC) and Physical Layer (PHY) specifications Amendment 8: Medium Access Control (MAC) Quality of Service Enhancements," IEEE Std 802.11e-2005 (Amendment to IEEE Std 802.11, 1999 Edition (Reaff 2003), pp.0_1-189, 2005.

[3] S. Chakrabarti and A. Mishra, "QoS issues in ad hoc wireless networks," IEEE Communications Magazine, vol. 39, no. 2, pp. 142-148, Feb. 2001.

[4] S. Chen and K. Nahrstedt, "Distributed quality-of-service routing in ad hoc networks," IEEE Journal on Selected Areas in Communications, vol.17, no. 8, pp. 1488-1505, Aug. 1999.

[5] S. Mao, S. Lin, S. S. Panwar, Y. Wang and E. Celebi, "Video transport over ad hoc networks: multistream coding with multipath transport," IEEE Journal on Selected Areas in Communications, vol. 21, no. 10, pp. 17211737, Dec. 2003.

[6] E. Setton, T. Yoo, X. Zhu, A. Goldsmith and B. Girod, "Cross-layer design of ad hoc networks for real-time video streaming," IEEE Wireless Communications, vol. 12, no. 4, pp. 59- 65, Aug. 2005.

[7] A. Ksentini, M. Naimi and A. Gueroui. "Toward an improvement of H.264 video transmission over IEEE 802.11e through a cross-layer architecture", IEEE Communications Magazine, vol. 44, no. 1, pp. 107-114, Jan. 2006.

[8] Iain Richardson, H.264 and MPEG-4 Video Compression: Video Coding for Next-generation Multimedia, Wiley, New York, NY, 2003.

[9] T. Wiegand and G. J. Sullivan, "The H.264/AVC Video Coding Standard," IEEE Signal Processing Magazine, vol. 24, no. 2, pp. 148 - 153, March 2007. 
[10] "Information technology- Telecommunications and information exchange between systems- Local and metropolitan area networks- Specific requirements- Part 11: Wireless LAN Medium Access Control (MAC) and Physical Layer (PHY) Specifications," ANSI/IEEE Std 802.11, 1999 Edition (R2003), pp. i-513, 2003.

[11] Charles E. Perkins and Elizabeth M. Royer. "Ad hoc On-Demand Distance Vector Routing." Proceedings of the 2nd IEEE Workshop on Mobile Computing Systems and Applications, New Orleans, LA, February 1999, pp. $90-100$

Firat Birlik received his B.S. degree in Computer Science and Engineering and M.S degree in Electronics Engineering both at Sabanci University, Istanbul, Turkey in 2005 and 2008, respectively. He is now working as fellow engineer for Airties Wireless Networks, Istanbul, Turkey.

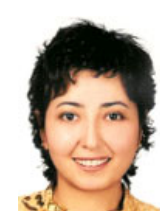

Özgür Gürbüz (M'92) received her B.S. and M.S degrees in Electrical and Electronics Engineering at Middle East Technical University, in 1992 and 1995, respectively. She received her Ph.D. degree in Electrical and Computer Engineering from Georgia Institute of Technology in 2000. From 2000 until 2002 she was a researcher and systems/algorithms engineer for Cisco Systems, in Wireless Access and Wireless Networking Business Units. She is currently an Assistant Professor in the Faculty of Engineering and Natural Sciences at Sabanci University. Her research interests are wireless communications and networks, in particular media access control, radio resource management, cross layer design, wireless networks with MIMO systems and smart antennas, and sensor network applications.

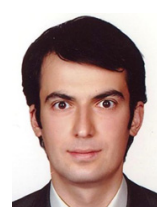

Ozgur Ercetin received the BS degree in electrical and electronics engineering from the Middle East Technical University, Ankara, Turkey, in 1995 and the MS and $\mathrm{PhD}$ degrees in electrical engineering from the University of Maryland, College Park, in 1998 and 2002, respectively. He was a research assistant at the University of Maryland and at HRL Laboratories, Malibu, California. Since 2002, he has been with the Faculty of Engineering and Natural Sciences, Sabanci University, Istanbul. His research interests are in the field of computer and communication networks with emphasis on fundamental mathematical models, architectures and protocols of wireless systems, sensor networks, high-speed Internet, and satellite communications. $\mathrm{He}$ is a member of the IEEE. 